\title{
을 Poly(ADP-ribosyl)ation regulates CTCF-dependent chromatin insulation
}

\author{
Wenqiang Yu ${ }^{1,6}$, Vasudeva Ginjala ${ }^{1,6}$, Vinod Pant ${ }^{1,6}$, Igor Chernukhin ${ }^{2,6}$, Joanne Whitehead ${ }^{1,6}$, \\ France Docquier ${ }^{2}$, Dawn Farrar ${ }^{2}$, Gholamreza Tavoosidana ${ }^{1}$, Rituparna Mukhopadhyay ${ }^{1}$, \\ Chandrasekhar Kanduri ${ }^{1}$, Mitsuo Oshimura ${ }^{3}$, Andrew P Feinberg ${ }^{4}$, Victor Lobanenkov ${ }^{5}$, Elena Klenova ${ }^{2}$ \\ \& Rolf Ohlsson ${ }^{1}$
}

Chromatin insulators demarcate expression domains by blocking the cis effects of enhancers or silencers in a positiondependent manner ${ }^{1,2}$. We show that the chromatin insulator protein CTCF carries a post-translational modification: poly(ADP-ribosyl)ation. Chromatin immunoprecipitation analysis showed that a poly(ADP-ribosyl)ation mark, which exclusively segregates with the maternal allele of the insulator domain in the $\mathrm{H} 19$ imprinting control region, requires the bases that are essential for interaction with $\mathrm{CTCF}^{3}$. Chromatin immunoprecipitation-on-chip analysis documented that the link between CTCF and poly(ADP-ribosyl)ation extended to more than 140 mouse CTCF target sites. An insulator trap assay showed that the insulator function of most of these CTCF target sites is sensitive to 3-aminobenzamide, an inhibitor of poly(ADP-ribose) polymerase activity. We suggest that poly(ADP-ribosyl)ation imparts chromatin insulator properties to CTCF at both imprinted and nonimprinted loci, which has implications for the regulation of expression domains and their demise in pathological lesions.

Poly(ADP-ribosyl)ation is traditionally associated with DNA repair and apoptosis ${ }^{4}$, but this view may be too limited ${ }^{5,6}$. For example, one of the poly(ADP-ribose) (PAR) polymerases, PARP-1, is associated both with formation of heterochromatin and with regions of high transcriptional activity in fruit flies ${ }^{7}$. To explore a potential correlation between poly(ADP-ribosyl)ation and expression domains in the mouse, we analyzed the allelic distribution of poly(ADP-ribosyl)ated protein complexes on the chromatin insulator at the H19 imprinting control region (ICR), which partitions expression domains in a parent of origin-specific manner ${ }^{8}$. We analyzed chromatin-immunoprecipitated DNA of fetal liver of M. musculus domesticus $\times$ M. musculus musculus intraspecific hybrid crosses by a PCR assay, which exploited a polymorphic BsmAI restriction site at the second CTCF target site?.
Only the maternally inherited allele was specifically captured using a specific antibody that detects polymers containing ten or more ADP-ribose units (Fig. 1a).

As the chromatin insulator protein CTCF is the only factor known to interact preferentially with the maternal H19 ICR allele in vivo ${ }^{3}$, we examined the interaction between poly(ADP-ribosyl)ated proteins and the $\mathrm{H} 19$ ICR with point-mutated CTCF target sites ${ }^{10}$. We carried out chromatin immunoprecipitation (ChIP) analysis of primary mouse fibroblast cultures, with the mutation inherited maternally or paternally, followed by PCR of the H19 ICR. The H19 ICR was associated with a poly(ADP-ribosyl)ation mark only if the wild-type allele was inherited maternally (Fig. 1b). Although this result suggested that the poly(ADP-ribosyl)ation mark of the maternal H19 ICR allele requires functional CTCF target sites, we could not rule out indirect effects from de novo methylation ${ }^{3}$. We therefore mixed equimolar amounts of plasmids containing the wild-type H19 ICR and plasmids containing the H19 ICR with mutations of CTCF target sites and transfected JEG3 cells with the mixture. ChIP assays on the protein-DNA complexes of the transfected plasmids showed that antibodies to CTCF and to PAR captured only the wild-type allele (Fig. 1c). As there is no de novo methylation of the episomal H19 ICR under these conditions (data not shown), poly(ADP-ribosyl)ated proteins associate to the H19 ICR exclusively through the CTCF target sites.

We explored the possibility that the poly(ADP-ribosyl)ated protein was identical to CTCF using in vitro poly(ADP-ribosyl)ating recombinant baculovirus CTCF (bvCTCF). After immunopurification, a substantial proportion of the total CTCF fraction was specifically retained (Fig. 2a). A modified western-blot protocol allowed us to resolve the default $(130 \mathrm{kDa})$ and poly (ADP-ribosyl)ated $(180 \mathrm{kDa})$ versions of bvCTCF (Fig. 2b). To confirm that CTCF is similarly poly(ADPribosyl)ated in vivo, we analyzed immunoprecipitated CTCF from several different sources (including MCF-7 breast cancer cells; Fig. 2e) by western blotting of nuclear extracts immunoprecipitated with a

${ }^{1}$ Department of Development \& Genetics, Evolution Biology Centre, Uppsala University, Norbyvägen 18A, S-752 36 Uppsala, Sweden. ${ }^{2}$ Department of Biological Sciences, Central Campus, University of Essex, Wivenhoe Park, Colchester, Essex C04 3SQ, UK. ${ }^{3}$ Department of Molecular and Cellular Biology, Tottori University, Tottori, Japan. ${ }^{4}$ Institute of Genetic Medicine, Johns Hopkins University School of Medicine, 1064 Ross, 720 Rutland Avenue, Baltimore, Maryland 21205 , USA. ${ }^{5}$ Molecular Pathology Section, Laboratory of Immunopathology, National Institute of Allergy and Infectious Diseases, National Institutes of Health, Bethesda, Maryland 20892, USA. ${ }^{6}$ These authors contributed equally to this work. Correspondence should be addressed to R.O. (Rolf.Ohlsson@ebc.uu.se). 
a

polyclonal antibody to CTCF. After immunoprecipitation with antibodies to CTCF and to PAR, we detected a 180-kDa band (Fig. 2c). We conclude that the poly(ADP-ribosyl)ated form of CTCF does exist in vivo and that it can be generated from recombinant CTCF by in vitro poly(ADP-ribosyl)ation.

To examine whether the poly(ADP-ribosyl)ated CTCF was able to interact with the H19 ICR, we used recombinant in vitro poly(ADPribosyl)ated and immunopurified bvCTCF (Fig. 2a) in mobility shift assays. Poly(ADP-ribosyl)ation of CTCF did not markedly interfere with its interaction with one of the CTCF target sites in the H19 ICR (Fig. 2d). Although we cannot rule out altered binding affinity of the poly(ADP-ribosyl)ated CTCF to its target sites, the mobility shift result is in contrast with previous reports on the ablation of DNA binding caused by the poly(ADP-ribosyl)ation of transcription factors such as YY1 (ref. 5). This difference might be due to the localization of key poly(ADP-ribosyl)ation sites, as the N-terminal portion, but not the 11-zinc-finger domain, of CTCF is the preferred in vitro target for PARP-1 (Fig. 2e).

We examined the generality of the link between CTCF and poly(ADP-ribosyl)ation using two approaches. First, a western-blot analysis of mouse neonatal tissues showed that the proportion of

Figure 2 CTCF is poly(ADP-ribosyl)ated. (a) CTCF can be poly(ADPribosyl)ated in vitro. The bvCTCF protein was poly(ADP-ribosyl)ated in vitro and subjected to affinity chromatography on a matrix with the immobilized antibodies to PAR. MW, molecular weight; PIS, preimmune serum. (b) Western-blot analysis of nonpoly(ADP-ribosyl)ated and poly(ADP-ribosyl)ated bvCTCF after optimization of the western-blotting conditions. MW, molecular weight. (c) Western-blot analysis of nonpoly(ADP-ribosyl)ated and poly(ADPribosyl)ated CTCF of the human breast cancer cell line MCF-7 after optimization of the western-blotting conditions. MW, molecular weight. (d) Mobility shift analysis shows that the affinity-purified, in vitro poly(ADPribosyl)ated CTCF retains its DNA binding capacity. The yCTCF lane depicts an EMSA analysis using Pichia recombinant CTCF. (e) The N-terminal domain of CTCF is a preferred target for poly(ADP-ribosyl)ation in vitro. Arrow indicates the position of the poly(ADP-ribosyl)ated $\mathrm{N}$-terminal domain of CTCF. C, C-terminal domain; N, N-terminal domain;

Zn, Zinc finger domain.
$180-\mathrm{kDa}$ CTCF relative to $130-\mathrm{kDa}$ CTCF varied in a tissue-specific manner (Fig. 3a). Notably, the 180-kDa poly(ADP-ribosyl)ated CTCF was abundant in heart and muscle but scarce in neonatal liver and brain, suggestive of tissue-specific differences in the turnover of the poly(ADP-ribosyl)ation mark. Because the poly(ADP-ribosyl)ation mark might, in some instances, not relate to the pool of CTCF but be a specific feature of the DNA-bound CTCF complexes, we used a second strategy: ChIP on chip analysis, exploiting CTCF target site library microarrays. Figure $\mathbf{3} \mathbf{b}$ shows the hybridization images and Figure $3 \mathrm{c}$ shows a scatter plot analysis comparing the hybridization signals clone by clone. This analysis indicated that $78 \%$ of the CTCF target sites of the mouse liver library were immunoprecipitated by the antibodies to PAR and to CTCF.

Whereas the data in Figure 3a suggest that only a fraction of CTCF is poly(ADP-ribosyl)ated before its association to target sites in some tissues, the data in Figure $\mathbf{3 b}, \mathbf{c}$ show that there is a strong correlation between CTCF occupancy and the presence of a poly(ADP-ribosyl)ation mark. The exceptions to this rule could be due to the fact that PAR marks involving fewer than ten units are not detected by the antibody to PAR. Taken together, the results indicate that a mechanism underlying the formation or the extension of the PAR mark exists
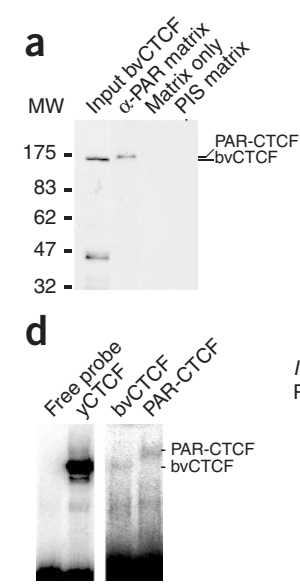

b c

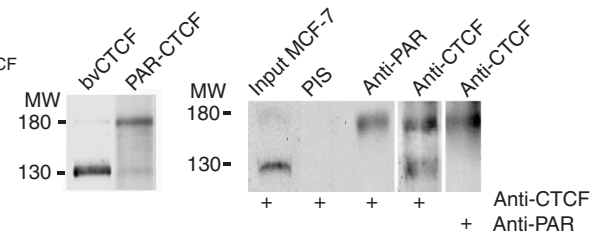

e

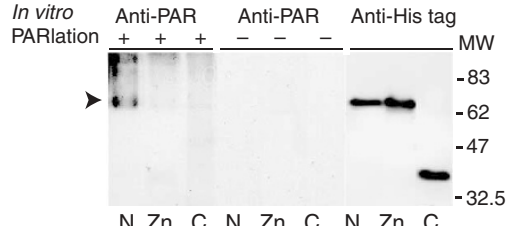


Figure 3 Extensive overlap in the patterns of poly(ADP-ribosyl)ation marks and CTCF occupancy. (a) A western-blot analysis of total representation of nonpoly(ADP-ribosyl)ated (130 kDa) and poly(ADP-ribosyl)ated CTCF $(180 \mathrm{kDa})$ in various mouse neonatal tissues. $\mathrm{MW}$, molecular weight. $(\mathbf{b}, \mathbf{c})$ ChIP probes, derived from neonatal liver and enriched in sequences interacting in vivo with CTCF or poly(ADP-ribosyl)ated proteins, were hybridized to microarrays of a library of CTCF target sites. (b) A selection of relevant array images of ChIP on chip hybridizations. (c) A scatter-plot analysis (in log2 scale) comparing the PAR and CTCF hybridization signals from these images. Control ChIP samples, obtained by using normal serum, showed no detectable signal in either instance (data not shown).

at some individual sites only after the CTCF-DNA complex has been formed. This possibility is supported by the observation that CTCF target sites can be relocated to the nucleoli, which are enriched in PARP-1, through a CTCF-nucleophosmin interaction ${ }^{11}$. Therefore, the tethering of CTCF target sites to the nucleolus might be essential for de novo poly(ADP-ribosyl)ation of CTCF and its insulator function.

To investigate this possibility, we used an insulator trap assay to examine a functional link between poly(ADP-ribosyl)ation and CTCFdependent chromatin insulator function. This assay ${ }^{12}$ depends on the ability of the candidate sequence to protect the toxin-A gene from the SV40 enhancer in an episomal context. We transfected JEG-3 cells with the plasmid mixture ligated with or without the H19 ICR and scored the number of surviving colonies, which is directly proportional to insulator strength. Under these conditions, there is no detectable integration of the episomal constructs into the genome $e^{9,13-15}$. Figure 4a outlines the toxin vectors used. H19 ICR insulator function is sensitive to 3-aminobenzamide (3-ABA), a general inhibitor of PAR polymerases, as the numbers (Fig. $\mathbf{4 b}$ ) and sizes (data not shown) of colonies surviving treatment with 3-ABA were substantially reduced in cells without the H19 ICR in the insulator position of the toxin-A vector. We ruled out the possibility that double selection by hygromycin and 3-ABA adversely affected the survival of the cells: in the absence of the toxin-A reporter gene, there was no marked difference in cell survival after treatment with hygromycin versus hygromycin and 3-ABA (Fig. 4b). Figure 4c documents the efficiency of the 3-ABA treatment, which rapidly eliminated the PAR content of H19 ICR chromatin, whereas CTCF association remained intact.

We inserted the entire amplified CTCF target site library into the insulator position of the pREPtoxA vector, between the toxin-A promoter and the SV40 enhancer. We used amplification primers with $X h o I$ and $K p n I$ restriction sites to prevent the generation of vectors with multiple inserts. After transfecting cells with the ligation mixture and selecting with hygromycin for three weeks, we scored for surviving cell colonies, as described above. Treatment with 3-ABA reduced the number of surviving colonies by $>70 \%$ (Fig. 4b). This result could indicate, by analogy to the effect on the H19 ICR, that there is a gradual and similar loss of insulator function for all the insulator segments. Alternatively, the effect might be specific for some insulators.

To discriminate between these possibilities, we amplified the insulator DNA segments by flanking primers from DNA of pooled surviving cell clones and then hybridized it to the CTCF target site library microarray. We visualized the resulting patterns by comparing the hybridization signal ratios of samples treated with 3-ABA versus those of untreated samples (Fig. 4d). The lower the insulator index ratio is, the more sensitive the insulator is to 3-ABA. The hybridization signal in the control insulator trap assay, which is a direct correlate of insulator strength ${ }^{12}$, served as an internal reference. The scatter-plot analysis indicated that most of the insulator sequences were sensitive

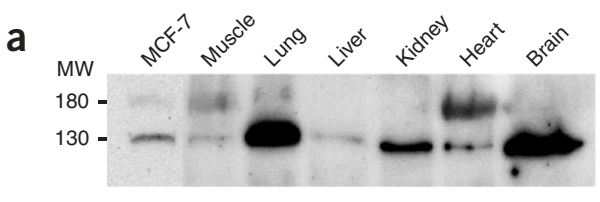

b

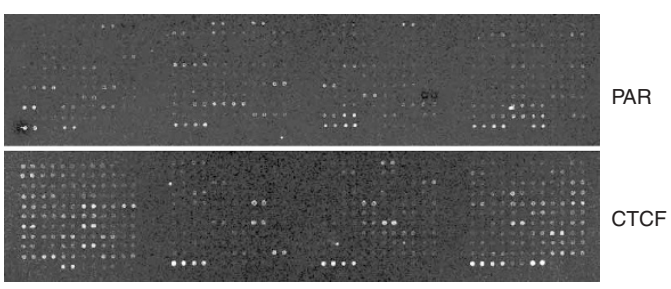

C

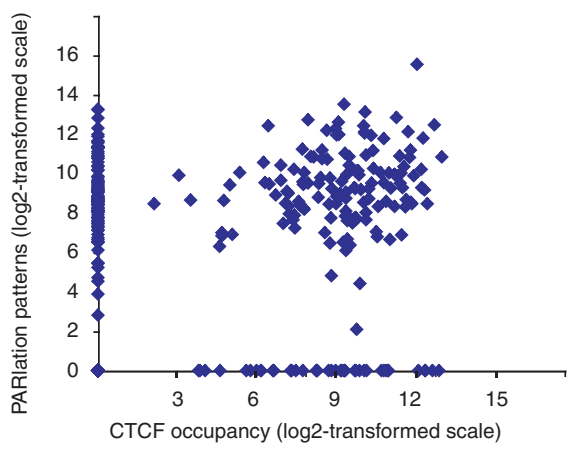

to 3-ABA (Fig. 4d). By plotting the standard deviation of the insulator index of binned data points across the control insulator axis, we visualized the high variation in 3-ABA sensitivity of weak insulators relative to strong insulators (Fig. 4e). This trend suggests that the 3-ABA sensitivity of the insulator property is common to all CTCF target sites.

As the endogenous insulator of the maternal H19 ICR allele insulates the upstream Igf2 from downstream enhancers, we examined the effect of 3-ABA treatment on allelic IGF2 expression patterns using mouse cells carrying human chromosome 11 of maternal or paternal origin $^{16}$. RNase protection analysis showed that the repressed status of the maternal IGF2 allele was lost in cells treated with 3-ABA (Fig. 4f). This result was paralleled by a reduction of the poly(ADP-ribosyl)ation mark at the H19 ICR (Fig. 4g), strengthening the link between a poly(ADP-ribosyl)ation-dependent chromatin insulator and the repression of the maternal IGF2 allele.

We conclude that there is a genome-wide and potentially contextdependent link between CTCF, poly(ADP-ribosyl)ation and chromatin insulator function. Although there is no report published on epigenetic lesions in mice with a targeted deletion of Adprt (encoding PARP-1), a short form of PARP-1 is constitutively expressed in $A d p r t^{-1-}$ cells and enzymatically active in a DNA damage-independent manner ${ }^{17}$. Moreover, the known PAR polymerase gene family encoding 3-ABA-sensitive polymerases is continuously expanding, suggesting that there is a certain degree of redundancy of constitutively expressed PAR polymerase enzymatic functions ${ }^{5}$. The polymerases do not constitute the whole picture, however, as poly(ADP-ribosyl)ation marks are rapidly turning over ${ }^{18}$. The macro domain of histone macroH2A, which covers only the inactive $\mathrm{X}$ chromosome ${ }^{19}$, has been proposed to enzymatically degrade $\mathrm{PAR}^{20}$. Accordingly, one function of macroH2A might be to erase chromatin insulator functions to facilitate the spreading of silencing along the chromatin fiber 
a
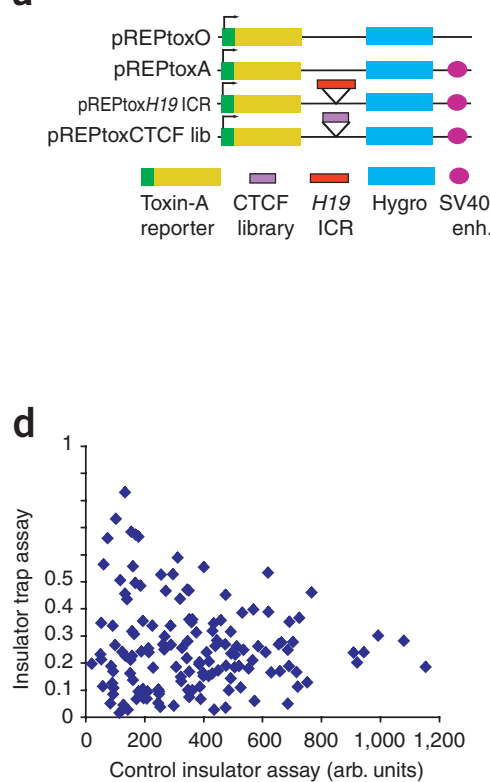

b

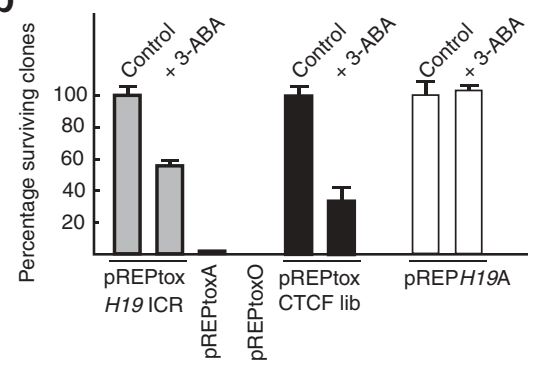

C

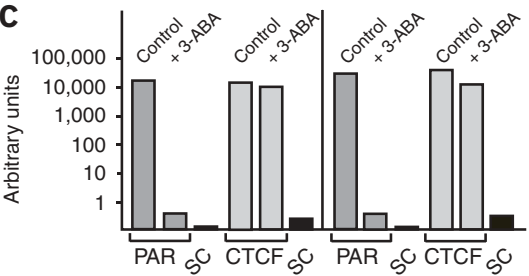

f

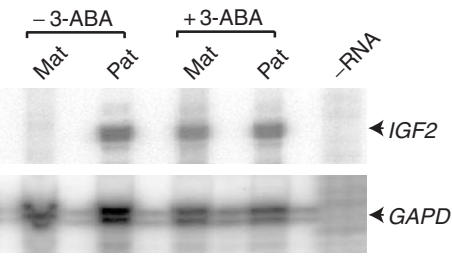

e

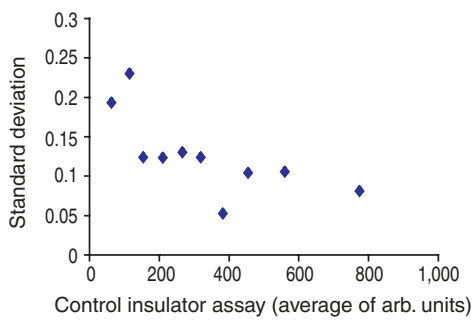

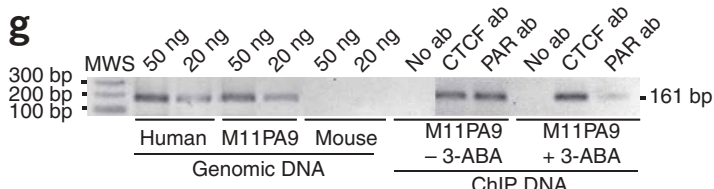

Figure 4 The insulator trap assay and 3-ABA sensitivity of chromatin insulator functions. (a) Schematic maps of the different pREPtox vectors. (b) The number of surviving cell clones in the presence or absence of 3-ABA. (c) Inhibition of PAR polymerases directly affects the PAR content of the H19 ICR. The specific representation of $H 19$ ICR sequences in each of the ChIP samples was determined by real-time PCR analysis. Two independent experiments are shown. SC, serum control. (d) A scatter-plot analysis of insulator strength in the presence or absence of 3-ABA, as determined from the microarray analysis. (e) A standard deviation analysis indicating that lower-strength insulators have a higher deviation from average than higher-strength insulators.

(f) Poly(ADP-ribosyl)ation and the imprinted state. IGF2 expression levels were analyzed by RNase protection of RNA isolated from mouse-human cell hybrids, untreated (control) or treated with 3-ABA. (g) ChIP assays indicate that the activation of the maternal IGF2 allele in M11PA9 cells was paralleled by a strong reduction in the poly(ADP-ribosyl)ation mark of the H19 ICR of human origin. MWS, molecular weight standard.

during X-chromosome inactivation. These considerations offer new perspectives also in cancer, as an unscheduled change in the dynamic equilibrium of constitutive PAR marks might lead to loss of CTCFdependent chromatin insulator functions, with ensuing pathological lesions in expression domains and networks.

\section{DOMETHODS}

Mice. We used M. m. musculus (CZECH II, Jackson Laboratories) and M. $m$. domesticus (NMRI strain) mice to create intraspecific $\mathrm{F}_{1}$ hybrid $M . m$. domesticus $\times M . m$. musculus or $M . m$. musculus $\times M . m$. domesticus conceptuses (where the mother's strain is given first and the father's strain second). We crossed the $142^{\star}$ strain with three of the four CTCF target sites mutated in a $129 / \mathrm{SvJ}$ background ${ }^{10}$ with the recombinant SD7 strain (gift from W. Reik; Babraham Institute, Cambridge, UK) ${ }^{21}$. We collected fetal livers at $16.5 \mathrm{~d}$ post coitum and neonatal tissues from 2-d-old conceptuses. All animal experiments were approved by the local ethical committee at Uppsala.

Cell cultures and transfections. We established primary cultures of fetuses (e13; $142^{*} \times$ SD7 or SD7 $\times 142^{*}$ crosses) and propagated them in Dulbecco's modified Eagle medium (Gibco BRL) supplemented with $10 \%$ fetal bovine serum, penicillin/streptomycin (Gibco) and glutamax. We maintained the JEG-3 cell line as described ${ }^{22}$. We added 3-ABA (Sigma) to the culture medium $28 \mathrm{~h}$ after transfection at a final concentration of $8 \mathrm{mM}$. We collected the cells after $14 \mathrm{~h}$ of exposure to 3-ABA. We maintained the M11PA9 maternal and P11PA9 paternal cell lines ${ }^{16}$ in Dulbecco's modified Eagle medium (Gibco BRL) supplemented with $10 \%$ fetal bovine serum, penicillin/streptomycin (Gibco) and Blasticidin S (CAYLA). We treated the maternal and paternal cell lines with $8 \mathrm{mM}$ of 3-ABA for $48 \mathrm{~h}$. We collected total RNA using the Qiagen RNA extraction kit. We carried out transfections using the FuGene 6 transfection reagent (Roche) with equimolar amounts of the plasmid DNAs ( $5 \mu \mathrm{g}$ for the basal promoter constructs) and $0.5 \mu \mathrm{g}$ of control plasmid (pREP9-RFP).
Chromatin immunopurification assay. We collected cells and crosslinked them with formaldehyde as described ${ }^{3}$. We immunopurified the DNA-protein complexes using antibody to PAR polymer (Trevigen) and protein A 4 Fast Flow Sepharose beads (Amersham Biosciences) and then amplified the immunopurified DNA from mouse fetal liver by PCR using BsmAI forward and reverse primers (sequences available on request). The PCR conditions were $95{ }^{\circ} \mathrm{C}$ for $5 \mathrm{~min}$; six cycles of $94^{\circ} \mathrm{C}$ for $1 \mathrm{~min}, 57^{\circ} \mathrm{C}$ for $1 \mathrm{~min}$ and $72{ }^{\circ} \mathrm{C}$ for $1 \mathrm{~min} ; 24$ cycles of $94^{\circ} \mathrm{C}$ for $30 \mathrm{~s}, 57^{\circ} \mathrm{C}$ for $30 \mathrm{~s}$ and $72{ }^{\circ} \mathrm{C}$ for $30 \mathrm{~s}$; and $72{ }^{\circ} \mathrm{C}$ for $3 \mathrm{~min}$. We amplified the immunopurified DNA from the mutated or wildtype H19 ICR allele by PCR using S2-Par forward primer and Meth-Dn reverse primer (sequences available on request). One of the primers was labeled with $\mathrm{P}^{32}-\gamma$-ATP (Amersham Biosciences). The PCR conditions were $95^{\circ} \mathrm{C}$ for $5 \mathrm{~min}$; 25 cycles of $94{ }^{\circ} \mathrm{C}$ for $30 \mathrm{~s}, 54{ }^{\circ} \mathrm{C}$ for $40 \mathrm{~s}$ and $72{ }^{\circ} \mathrm{C}$ for $40 \mathrm{~s}$; and $72{ }^{\circ} \mathrm{C}$ for $5 \mathrm{~min}$. We visualized the PCR products on a $6 \%$ acrylamide gel and analyzed them with a Fuji-FLA 3000 Phosphorimager or exposed them on Kodak BioMax-MS X-ray film.

Real-time PCR analysis of ChIP samples. We transfected JEG-3 cells with the pB-GFP plasmid, treated some of them with $8 \mathrm{mM}$ of 3-ABA after transfection and left others untreated. We carried out ChIP with antibodies to PAR (Trevigen) and to CTCF (Upstate Biotechnologies). We diluted DNA samples to $10 \mathrm{ng} \mu \mathrm{l}^{-1}$ and carried out real-time PCR analysis using an ABI PRISM-7700 cycler (Applied Biosystems), Taqman Gold Universal Master mix (PerkinElmer) and TmanS1-For- and TmanS1-Rev- primers (sequences available on request). PCR conditions were $95{ }^{\circ} \mathrm{C}$ for $10 \mathrm{~min}$ followed by 40 cycles at $95{ }^{\circ} \mathrm{C}$ for $15 \mathrm{~s}$ and $60{ }^{\circ} \mathrm{C}$ for $1 \mathrm{~min}$.

Analysis of IGF2 expression and poly(ADP-ribosyl)ation mark. We extracted RNA from the mouse-human hybrid M11PA9 and P11PA9 cell lines and analyzed it by RNase protection using the Ambion RPA III kit. We cut a 558-bp HinfI-PstI human IGF2 cDNA insert ${ }^{23}$ with XhoI and used it as the template to generate an IGF2 antisense riboprobe (145 bases; SP6 polymerase $)^{24}$. We carried out the ChIP assay of M11PA9 cells treated with 3-ABA using an 
antibody to PAR as described above. We analyzed the region 2 human H19 ICR, propagated in mouse cells, by PCR amplification as described ${ }^{25}$.

Recombinant CTCF and poly(ADP-ribosyl)ation. We analyzed CTCF expression in the baculovirus system and expression of the N-terminal, zinc finger and C-terminal domains of CTCF in a bacterial system as previously described $^{26}$. We prepared Pichia recombinant CTCF, a gift from A Vostrov and W. Quitschke (Department of Psychiatry and Behavioral Science, State University of New York at Stony Brook, New York, USA), as described ${ }^{27}$. The bvCTCF protein and the three CTCF domains were poly(ADP-ribosyl)ated using a published protocol ${ }^{28}$ with modifications. We incubated $\sim 50 \mathrm{pM}$ of protein in $200 \mu \mathrm{l}$ of a reaction buffer containing $50 \mathrm{mM}$ Tris-HEPES buffer $(\mathrm{pH}$ 8.0), $0.15 \mathrm{mM} \mathrm{KCl}, 10 \mathrm{mM} \mathrm{MgCl}, 10 \mathrm{mM}$ 2-mercaptoethanol, $200 \mu \mathrm{M}$ $\beta \mathrm{NAD}^{+}, 200 \mathrm{ng}$ of calf thymus DNA pretreated with DNase I and $500 \mathrm{ng}$ of bovine PAR polymerase (Alexis Biochemicals) for $1 \mathrm{~h}$ at $30^{\circ} \mathrm{C}$. We purified the poly(ADP-ribosyl)ated CTCF protein using a matrix with immobilized antibodies to PAR. We mixed $50 \mu$ lof antibody to PAR-sepharose conjugate with a bvCTCF protein sample diluted to $0.5 \mathrm{ml}$ with immunoprecipitation buffer (50 mM Tris-HEPES buffer ( $\mathrm{pH}$ 8.0), $0.15 \mathrm{M} \mathrm{NaCl}, 2 \mathrm{mM}$ EDTA and $0.1 \%$ Triton X-100) and incubated the mixture for $3 \mathrm{~h}$ at $4{ }^{\circ} \mathrm{C}$ on a rotating platform. After washing, we eluted the protein with $0.1 \mathrm{M} \mathrm{NaH}_{2} \mathrm{PO}_{4}$ (pH 4.0), $20 \mathrm{mM}$ 2-mercaptoethanol and $7 \mathrm{M}$ urea and renatured it by dialysis against $10 \mathrm{mM}$ HEPES buffer- $\mathrm{KOH}$ ( $\mathrm{pH} 7.0$ ), $0.3 \mathrm{M} \mathrm{KCl}$ and $10 \mathrm{mM}$ 2-mercaptoethanol containing $10 \%$ glycerol.

Western-blot analysis. We prepared extracts from tissues as follows. We solubilized frozen tissue sections in lysis buffer $(20 \mathrm{mM}$ Tris buffered with HEPES buffer ( $\mathrm{pH}$ 8.0), $2 \mathrm{mM}$ EDTA, $0.5 \mathrm{M} \mathrm{NaCl}, 0.5 \%$ sodium deoxycholate, $0.5 \%$ Triton X-100, $0.25 \mathrm{M}$ sucrose, $50 \mathrm{mM}$ 2-ME and a cocktail of inhibitors; O Roche) at approximately $5 \mathrm{mg}$ of tissue per $\mathrm{ml}$ buffer and homogenized them. We then incubated the extracts on ice for $20 \mathrm{~min}$, filtered them to remove unresolved tissue particles, centrifuged them at 14,000 r.p.m. at $4{ }^{\circ} \mathrm{C}$, collected the supernatants, mixed them with SDS-loading buffer (1/1, vol/vol), heated them for $5 \mathrm{~min}$ at $95^{\circ} \mathrm{C}$ and resolved them in SDS-PAGE. We optimized the conditions to resolve poly(ADP-ribosyl)ated and nonpoly(ADP-ribosy1)ated forms of CTCF as follows. We resolved the samples in 6.5\% SDS-PAGE and then subjected the gel to treatment for $15 \mathrm{~min}$ in an SDS running buffer with $1 \%$ methanol before blotting to improve the transfer of the poly(ADPribosyl)ated form of CTCF. We electroblotted the gels onto Immobilon P (2) polyvinylidene difluoride filters (Millipore), probed membranes with the primary antibodies (at 1:1,000 dilution for the antibody to PAR $10 \mathrm{H}$ (Trevigen/Chemicon) and 1:1,000 dilution for the polyclonal antibody to CTCF (Abcam) ) and then incubated the samples with the secondary antibodies to mouse and to rabbit, respectively. We developed the membranes with the ECL kit (Amersham Biosciences).

Band-shift assay. We end-labeled a 120-bp fragment containing the fourth CTCF site in the H19 $\mathrm{ICR}^{29}$ using T4-PNK (New England Biolabs) and ${ }^{32} \mathrm{P}-\gamma$ ATP (Amersham Biosciences). We carried out the band-shift assay, using full-length bvCTCF and affinity-purified poly(ADP-ribosyl)ated bvCTCF, as described ${ }^{30}$.

ChIP on chip hybridization analysis. We amplified the ChIP DNA specimens by a two-step PCR method ${ }^{12}$. We prepared the probe using CyScribe PostLabelling kit (Cy Dye Post-labelling Reactive Dye Pack, Amersham Biosciences). We pooled probes and precipitated them with $100 \mu \mathrm{g}$ of Cot-1 DNA (Clontech). We dissolved the labeled DNA in water and hybridization solution (GlassHyb Hybridization solution, Clontech), denatured it and incubated it at $45^{\circ} \mathrm{C}$ for $2 \mathrm{~h}$. We hybridized and washed microarrays in accordance with the GlassHyb Hybridization Solution kit User Manual (Clontech). We scanned the slides using a Scanarray 4000 and analyzed them with Quantarray version 3.0 (Packard Biosciences). Local background and signals from negative controls were subtracted.

Insulator trap assay. The pREPtoxA vector contains a multiple cloning site flanked by two reporter genes ${ }^{12}$. We scored the Diphtheria toxin- $\mathrm{A}$ gene for insulator and silencer properties and the hygromycin gene only for silencing activity. We used the multiple cloning site to insert the entire
CTCF library with aid of primers Xho1-T7, Kpn1-Sp6, Kpn1-T7 and Xho1-Sp6 (sequences available on request). We transfected the JEG-3 cell line with the entire ligation mixture and then selected clones against hygromycin $\left(150 \mu \mathrm{g} \mathrm{ml}^{-1}\right)$ for 2-3 weeks. We prepared genomic DNA from the surviving clones using the Wizard Genomic DNA Purification kit (Promega). We amplified the inserts by PCR using the above primers, labeled them and hybridized them to the CTCF target site library microarray as above.

\section{ACKNOWLEDGMENTS}

We thank A. Mattsson for expert technical assistance, H. Smith and M. O'Farrell for help with the in vitro poly(ADP-ribosyl)ation assay, S. Shamsuddin for providing the domains of CTCF expressed in bacterial system, P. Hassa for helpful comments, A. Vostrov and W. Quitschke for the recombinant CTCF from Pichia and A. Isaksson and the Wallenberg microarray platform at the Rudbeck Laboratory for their assistance. This work was supported by the Swedish Science Research Council (V.R., R.O.), the Swedish Cancer Research Foundation (C.F., R.O.), the Swedish Pediatric Cancer Foundation (B.C.F., R.O.), the Lundberg Foundation (R.O.), the Wallenberg Foundation (R.O.), the Juvenile Diabetes Research Foundation International (R.O.), the Breast Cancer Campaign (F.D., E.K.), PhD Studentship from the University of Essex (D.F.), Research Promotion Fund from the University of Essex (I.C., F.D., E.K.) and the Association for International Cancer Research (I.C., E.K.).

\section{COMPETING INTERESTS STATEMENT}

The authors declare that they have no competing financial interests.

Received 30 June; accepted 6 August 2004

Published online at http://www.nature.com/naturegenetics/

1. Bell, A.C., West, A.G. \& Felsenfeld, G. Insulators and boundaries: versatile regulatory elements in the eukaryotic genome. Science 291, 447-450 (2001).

2. Ohlsson, R., Renkawitz, R. \& Lobanenkov, V. CTCF is a uniquely versatile transcription regulator linked to epigenetics and disease. Trends Genet. 17, 520-527 (2001).

3. Kanduri, C. et al. Functional interaction of CTCF with the insulator upstream of the H19 gene is parent of origin-specific and methylation-sensitive. Curr. Biol. 10, 853856 (2000).

4. Ziegler, M. \& Oei, S.L. A cellular switch: poly(ADP-ribosy)lation stimulates DNA repair and silences transcription. Bioessays 23, 543-548 (2001).

5. Smith, S. The world according to PARP. Trends Biochem. Sci. 26, 174-179 (2001).

6. Kraus, W. \& Lis, J. PARP goes transcription. Cell 113, 677-683 (2003).

7. Tulin, A., Stewart, D. \& Spradling, A. The Drosophila heterochromatic gene encoding poly(ADP-ribose) polymerase (PARP) is required to modulate chromatin structure during development. Genes Dev. 16, 2108-2119 (2002).

8. Thorvaldsen, J.L., Duran, K.L. \& Bartolomei, M.S. Deletion of the H19 differentially methylated domain results in loss of imprinted expression of H19 and Igf2. Genes Dev. 12, 3693-3702 (1998).

9. Kanduri, C. et al. The $5^{\prime}$-flank of the murine $\mathrm{H} 19$ gene in an unusual chromatin conformation unidirectionally blocks enhancer-promoter communication. Curr. Biol. 10, 449-457 (2000)

10. Pant, V. et al. The nucleotides responsible for the direct physical contact between the chromatin insulator protein CTCF and the $\mathrm{H} 19$ imprinting control region manifest parent of origin-specific long-distance insulation and methylation-free domains. Genes Dev. 17, 586-590 (2003).

11. Yusufzai, T., Tagami, H., Nakatani, Y. \& Felsenfeld, G. CTCF tethers an insulator to subnuclear sites, suggesting shared insulator mechanisms across species. Mol. Cell 13, 291-298 (2004).

12. Mukhopadhyay, R. et al. The binding sites for the chromatin insulator protein CTCF map to DNA methylation-free domains genomewide. Genome Res. 14, 1594-1602 (2004).

13. Holmgren, C. et al. CpG methylation regulates the Igf2/H19 insulator. Curr. Biol. 11, 1128-1130 (2001).

14. Kanduri, C., Kanduri, M., Thakur, N., Pfeifer, S. \& Ohlsson, R. The kinetics of deregulation of expression by de novo methylation of the H19 imprinting control region in cancer cells. Cancer Res. 62, 4545-4548 (2002).

15. Kanduri, C. et al. A differentially methylated imprinting control region within the Kcnq1 locus harbours a methylation-sensitive chromatin insulator. J. Biol. Chem. 277 , 18106-18110 (2002).

16. Horike, S. et al. Targeted disruption of the human LIT1 locus defines a putative imprinting control element playing an essential role in Beckwith-Wiedemann syndrome. Hum. Mol. Genet. 9, 2075-2083 (2000).

17. Sallmann, F.R., Vodenicharov, M.D., Wang, Z.Q. \& Poirier, G.G. Characterization of SPARP-1. An alternative product of PARP-1 gene with poly(ADP-ribose) polymerase activity independent of DNA strand breaks. J. Biol. Chem. 275, 15504-15511 (2000). 
18. Davidovic, L., Vodenicharov, M., Affar, E. \& Poirier, G. Importance of poly(ADP-ribose) glucohydrolase in the control of poly(ADP-ribose) metabolism. Exp. Cell Res. 268, 713 (2001).

19. Constanzi, C. \& Pehrson, J. Histone macroH2A1 is concentrated in the inactive $X$ chromosome in female mammals. Nature 393, 599-601 (1998).

20. Allen, M., Buckle, A., Cordell, S., Lowe, J. \& Bycroft, M. The crystal structure of AF1521 a protein from Archaeoglobus fulgidus with homology to the non-histone domain of macroH2A. J. Mol. Biol. 330, 503-511 (2003).

21. Dean, W. et al. Altered imprinted gene methylation and expression in completely ES cell-derived mouse fetuses: association with abberant phenotypes. Development 125 , 2273-2282 (1998).

22. Franklin, G. et al. The human PDGF-B gene is regulated by cell type-specific activator and suppressor elements in the first intron. EMBO J. 10, 1365-1373 (1991).

\& 23. Ohlsson, R., Holmgren, L., Glaser, A., Szpecht, A. \& Pfeifer-Ohlsson, S. Insulin-like EMBO J. 8, 1993-1999 (1989).

24. Li, Y.-M. et al. The H19 transcript is associated with polysomes and may regulate IGF2 expression in trans. J. Biol. Chem. 273, 28247-28252 (1998).
25. Lynch, C., Tycko, B., Bestor, T. \& Walsh, C. Reactivation of a silenced $H 19$ gene in human rhabdomyosarcoma by demethylation of DNA but not histone hyperacetylation. Mol. Cancer. 1, 2 (2002)

26. Chernukhin, I. et al. Physical and functional interaction between two pluripotent proteins the Y-box DNA/RNA-binding factor, YB-1, and the multivalent zinc finger factor, CTCF. J. Biol. Chem. 275, 29915-29921 (2000).

27. Quitschke, W.W., Taheny, M.J., Fochtmann, L.J. \& Vostrov, A.A. Differential effect of zinc finger deletions on the binding of CTCF to the promoter of the amyloid precursor protein gene. Nucleic Acids Res. 28, 3370-3378 (2000).

28. Miranda, E., Dantzer, F., O'Farrell, M., de Murcia, G. \& de Murcia, J. Characterisation of a gain-of-function mutant of poly(ADP-ribose) polymerase. Biochem. Biophys. Res. Commun. 212, 317-325 (1995).

29. Kanduri, M. et al. Multiple nucleosome positioning sites regulate the CTCF-mediated insulator function of the $\mathrm{H} 19$ imprinting control region. Mol. Cell. Biol. 22, 33393344 (2002).

30. Klenova, E. et al. CTCF, a conserved nuclear factor required for optimal transcriptional activity of the chicken c-myc gene, is an 11-Zn-finger protein differentially expressed in multiple forms. Mol. Cell. Biol. 13, 7612-7624 (1993). 